\title{
Tigecycline Susceptibility in Multidrug Resistant Acinetobacter Isolates from Turkey
}

\author{
FETHIYE FERDA YILMAZ ${ }^{1 *}$, HÜSEYIN TAŞLI ${ }^{1}$, SÜREYYA GÜL-YURTSEVER², AYÇA BÜYÜK ${ }^{1}$ \\ and MINE HOŞGÖR-LIMONCU ${ }^{1}$
}

\author{
${ }^{1}$ Ege University Faculty of Pharmacy, Dep. of Pharmaceutical Microbiology, İzmir, Türkiye \\ ${ }^{2}$ İzmir Katip Çelebi University, Atatürk Training and Research Hospital \\ Medical Microbiology Laboratory, İzmir, Türkiye.
}

Submitted 30 November 2012, revised 6 June 2013, accepted 15 July 2013

\begin{abstract}
The present study aimed to evaluate antimicrobial activity of tigecycline against 84 multidrug resistant (MDR) Acinetobacter spp. strains by disc diffusion and E-test methods. The results of disc diffusion test were compared according to two different interpretation ways. In addition, E-test results and the disc diffusion results that interpreted by both the methods were checked for compatibility. According to the disc diffusion test, 3 strains (3.57\%) were found resistant to tigecycline when considering breakpoints suggested by Food and Drug Administration (FDA). On the other hand, none of the strains was found resistant to the evaluation criteria recommended by Jones et al. (2007). Considering E-test results of tigecycline, $\mathrm{MIC}_{50}$ and $\mathrm{MIC}_{90}$ values of tigecycline for Acinetobacter spp. were $0.75 \mathrm{and} 1 \mathrm{mg} / \mathrm{l}$, respectively. Based on FDA defined breakpoints for Enterobacteriaceae, any resistant isolate was detected. In conclusion, although there are some differences in the results, tigecycline was found quite effective on Acinetobacter spp. isolates with reference to the both disc diffusion and the E-test methods.
\end{abstract}

Key words: Acinetobacter, antibiotic resistance, tigecycline

\section{Introduction}

Acinetobacter spp. is important opportunistic pathogen in nosocomial infections, which cause a wide range of clinical complications, such as pneumonia, septicemia and meningitis, especially in immunocompromised patients and intensive care units (ICUs). In recent years, new antibacterial agents are needed for the treatment of infections caused by multidrugresistant (MDR) Acinetobacter spp., including broadspectrum beta $(\beta)$-lactams, aminoglycosides, and fluoroquinolones (Falagas et al., 2008; Manchanda et al., 2010; Neonakis et al., 2011). Tigecycline was recently approved by the U.S. Food and Drug Administration (FDA) and the European Medicines Agency for the treatment of complicated skin and intra-abdominal infections. Tigecycline, the 9-tert-butyl-glycylamido derivative of minocycline, exhibits a broad-spectrum of activity against numerous pathogens, including Acinetobacter spp.. Like the tetracyclines, tigecycline binds to the $30 \mathrm{~S}$ subunit of bacterial ribosomes and inhibits protein synthesis by preventing the incorporation of amino acid residues into elongating peptide chains (Fraise, 2006; Neonakis et al., 2011; Peterson, 2008).

However, many researches indicated that there was a discrepancy in the susceptibility results of tigecycline against Acinetobacter spp. among different methods of testing such as broth microdilution, E-test, disc diffusion, and automated systems. Reference standard, broth microdilution testing serves as the method of comparison for the development and evaluation of alternative susceptibility testing methodologies. Recently, an E-test has been developed for the susceptibility testing of tigecycline. However, defined susceptibility breakpoints have not been declared thus far for A. baumannii in the latest issues of the Clinical and Laboratory Standarts Institute (CLSI) because of insufficient data about clinical usage of tigecycline (Liu et al., 2010; Neonakis et al., 2011; Shakoor et al., 2011). The unavailability of standard breakpoints of tigecycline leads to mistakes in categorization of MIC values and consequently gives rise to careless use of this antibiotic (Shakoor et al., 2011).

The first aim of the present study was to investigate the antimicrobial activity of tigecycline by disc diffusion

\footnotetext{
* Corresponding author: F.F. Yllmaz, Ege University Faculty of Pharmacy, Dep. of Pharmaceutical Microbiology, 35100 Bornova - İzmir, Türkiye; phone: +90 232 3112208; fax:+90 232 3885258; e-mail: fetferday@gmail.com
} 
method and the E-test for 84 clinical MDR Acinetobacter sp., and the second one was to compare the susceptibility assessment methods.

\section{Experimental}

\section{Material and Methods}

Bacteria. Between December 2009 and December 2010, 84 MDR Acinetobacter spp. isolates were collected from various clinical specimens at İzmir Katip Çelebi University, Atatürk Training and Research Hospital, Medical Microbiology Laboratory, Turkey. From the total 84 specimens obtained, 67 (80\%) were from ICUs. The isolates were identified and antimicrobial susceptibilities were determined by BD Phoenix System. MDR Acinetobacter spp. were defined as the isolates resistant to at least three classes of antimicrobial agents. The isolates were stored at $-80^{\circ} \mathrm{C}$, in the Brain Heart Infusion broth (Oxoid) supplemented with $10 \%$ glycerin.

Disc diffusion method. In vitro susceptibility of Acinetobacter spp. against tigecycline was determined by Kirby-Bauer disc diffusion method according to the CLSI guidelines, by using $15 \mu \mathrm{g}$ tigecycline discs (Becton Dickinson, USA) (CLSI). The results were evaluated by using disc diffusion breakpoints for Enterobacteriaceae proposed by FDA (susceptible $\geq 19 \mathrm{~mm}$ and resistant $\leq 14 \mathrm{~mm}$ ) and by Jones et al. (2007) (susceptible $\geq 16 \mathrm{~mm}$ and resistant $\leq 12 \mathrm{~mm}$ ). Escherichia coli ATCC 25922 was used as control strain.

E-test method. E-test Tigecycline gradient strips (AB Biodisc, Sweden; 0.016-256 $\mu \mathrm{g} / \mathrm{ml}$ ) were used according to CLSI guidelines and the MIC values were interpreted according to FDA defined breakpoints for Enterobacteriaceae (susceptible $\leq 2 \mathrm{mg} / \mathrm{l}$; intermediate $4 \mathrm{mg} / \mathrm{l}$; resistant $\geq 8 \mathrm{mg} / \mathrm{l}$ ) were applied in this study. MICs were read at $100 \%$ inhibition of growth. E. coli ATCC 25922 was used as the control strain.

Statistical analysis. Statistical analysis was performed using Minitab statistical software (Minitab Release $16^{\circ}$, State College, PA). For comparison of the evaluation criteria and antibiotic susceptibility tests results, $\mathrm{Z}$ test was employed. In all tests, differences were considered significant when $p<0.05$.

\section{Results}

This study showed that 3 Acinetobacter spp. strains (3.57\%) were resistant according to a disc diffusion method when considering breakpoints suggested by FDA. None of the strains was found resistant in the disc diffusion results according to Jones' criteria. Similarly, E-test method results showed no resistance in the Acinetobacter spp. strains. On the other hand, the susceptibility rate detected by the E-test method was statistically higher than the disc diffusion method according to both interpretation criteria $(\mathrm{p}<0.05)$ (Table I).

The tigecycline MIC range was found as $0.032-3 \mathrm{mg} / \mathrm{l}$ by E-test method. $\mathrm{MIC}_{50}$ and $\mathrm{MIC}_{90}$ values of tigecycline for Acinetobacter spp. were 0.75 and $1 \mathrm{mg} / \mathrm{l}$, respectively (Table II).

Table I

Comparing the tigecycline susceptibility of 84 Acinetobacter spp. isolates

\begin{tabular}{|l|l|c|c|c|}
\hline \multicolumn{1}{|c|}{ Methods } & $\begin{array}{c}\text { Evaluation } \\
\text { criteria }\end{array}$ & $\begin{array}{c}\text { S } \\
\mathrm{n}(\%)\end{array}$ & $\begin{array}{c}\mathrm{I} \\
\mathrm{n}(\%)\end{array}$ & $\begin{array}{c}\mathrm{R} \\
\mathrm{n}(\%)\end{array}$ \\
\hline Disc Diffusion & FDA $^{\mathrm{a}}$ & $46(54.76)$ & $35(41.67)$ & $3(3.57)$ \\
\hline & Jones criteria $^{\mathrm{b}}$ & $69(82.14)$ & $15(17.86)$ & - \\
\hline E-test & FDA $^{\mathrm{c}}$ & $83(98.81)$ & $1(1.19)$ & - \\
\hline
\end{tabular}

S: Susceptible, I: Intermediate, R: Resistant;

a FDA criteria for disc diffusion method: $S \geq 19 \mathrm{~mm}, \mathrm{R} \leq 14 \mathrm{~mm}$

${ }^{\mathrm{b}}$ Jones criteria for disc diffusion method: $\mathrm{S} \geq 16 \mathrm{~mm}, \mathrm{R} \leq 12 \mathrm{~mm}$

${ }^{c}$ FDA criteria for E-test method: $S \geq 2 \mu \mathrm{g} / \mathrm{ml}, \mathrm{R} \leq 8 \mu \mathrm{g} / \mathrm{ml}$

\section{Discussion}

Recently, some researches on in vitro activity of tigecycline against Acinetobacter showed a variability depending on the methodology used to determine susceptibility. For example, microdilution testing methodologies can show potent in vitro activity for tigecycline against MDR Acinetobacter spp., on the other hand the E-test can indicate high tigecycline resistance among clinical isolates (Kulah et al., 2009; Shakoor et al., 2011; Wang and Dowzicky, 2010). In this study, all the Acinetobacter sp. isolates were found to be susceptible to tigecycline although there were some differences in the results of the E-test and disc diffusion assays. Besides, E-test susceptibility results were supported by disc diffusion results when the recommendations by Jones et al.

Table II

MIC results of 84 Acinetobacter spp. isolates according to the E-test method

\begin{tabular}{|c|c|c|c|c|c|c|c|c|c|c|c|c|c|}
\hline & \multicolumn{13}{|c|}{ MIC values $(\mu \mathrm{g} / \mathrm{ml})$} \\
\hline & 0.032 & 0.064 & 0.094 & 0.125 & 0.19 & 0.25 & 0.38 & 0.5 & 0.75 & 1 & 1.5 & 2 & 3 \\
\hline $\mathbf{n}$ & 1 & 5 & 6 & 9 & 6 & 4 & 2 & 8 & 23 & 13 & 3 & 3 & 1 \\
\hline$(\%)$ & $(1.19)$ & $(6.0)$ & $(7.2)$ & $(10.8)$ & $(7.2)$ & $(4.8)$ & $(2.4)$ & $(9.5)$ & $(27.4)$ & $(15.5)$ & (3.6) & (3.6) & $(1.2)$ \\
\hline
\end{tabular}

$\mathrm{n}$ : Number of strain, $\mathrm{MIC}_{50}: 0.75 \mu \mathrm{g} / \mathrm{ml}, \mathrm{MIC}_{90}: 1 \mu \mathrm{g} / \mathrm{ml}$ 
(2007) were considered $(\mathrm{p}<0.05)$, and the MICs of the isolates did not exceed $3 \mathrm{mg} / \mathrm{l}$.

Wang and Dowzicky (2010) found low $\mathrm{MIC}_{90}$ values $(\leq 2 \mathrm{mg} / \mathrm{l})$ for tigecycline against Acinetobacter spp. isolates from blood samples, as similar studies published before. They also pointed out the deficiency of suggested breakpoints for tigecycline against Acinetobacter spp., thus comparing susceptibility studies based on different guides leads to confusing or even misleading results. When considereding the FDA breakpoint for Enterobacteriaceae, tigecycline inhibited at least $90.0 \%$ of isolates from all countries (Mendes et al., 2010).

In the east part of Turkey, of $71 \mathrm{~A}$. baumannii strains studied, 2 strains (3\%) were resistant, 35 strains (49\%) moderately susceptible, and 34 strains (48\%) susceptible against tigecycline according to the disk diffusion breakpoints proposed by FDA for Enterobacteriaceae and 1 strain (1\%) was resistant, 1 strain (1\%) moderately susceptible, and 69 strains (97\%) susceptible when considered the breakpoints according to Jones et al. Thus, it was asserted that the use of FDA criteria for tigecycline against Acinetobacter spp. was inaccurate, and also breakpoints suggested by Jones et al. should be supported by further investigations (Gülhan et al., 2009).

Bolmstrom et al. (2007) showed that the tigecycline E-test gradient method was as accurate as the reference methods. In addition, the error rates were very low. Hope et al. (2007) determined that tigecycline E-tests were shown to have good correlation with agar dilution MICs. However, Thamlikitkul et al. (2007) indicated that there was a discrepancy in the susceptibility results of tigecycline against Acinetobacter spp. among different methods of testing. The MICs determined by the E-test were usually four-fold higher than those determined by the broth microdilution method. Similarly, Pillar et al. (2008) observed a four-fold increase in $\mathrm{MIC}_{90}$ value among tested A. baumannii by E-test relative to broth microdilution test and noted a difference between the two testing methodologies. Liu et al. (2010) compared the results of E-test and broth microdilution method for tigecycline susceptibility testing of 393 A. baumannii isolates collected from 19 hospitals in Taiwan. E-test results showed an agreement in $76.6 \%$ of the strains when compared with the broth microdilution method. According to the results they declared that the E-test is not ideal as a substitute for broth microdilution testing in determining the MICs of tigecycline against A. baumannii isolates. Zarate et al. (2010) assayed in parallel by the broth microdilution, agar dilution, and disc diffusion method in $60 \mathrm{MDR}$ Acinetobacter spp. isolates obtained from hospitalized patients at two teaching hospitals in Argentina. A comparative analysis between methods by scattergram correlation and analysis of MICs and diameter zones around the disk was performed. They found a positive lineal correlation between the methodolo- gies. Using the FDA Enterobacteriaceae susceptibility breakpoint for tigecycline, an acceptable minor error rate was observed by agar dilution and broth microdilution, but an unacceptable error by the disc diffusion method. In another study from Pakistan (Shakoor et al., 2010), in vitro activity of tigecycline against 100 Acinetobacter spp. were determined by E-test and the MICs were interpreted according to both the BSAC and FDA breakpoints. Their data has changed significantly from $94 \%$ sensitive to $79 \%$ non-susceptible (intermediate or resistant), thus the authors underlined the importance of requirement universally compliant breakpoints for tigecycline against Acinetobacter spp.

\section{Conclusions}

Management of Acinetobacter spp. infections is difficult due to the emergence of isolates with multipledrug resistance. Thus, it is necessary to evaluate new molecules that are potentially useful against Acinetobacter spp. Tigecycline is seems to be a good choice for succeed in therapy. It is also an important to monitor the increase of the resistance in the microorganisms during the usage of tigecycline for treatment. The development and validation of reliable methods for antimicrobial susceptibility testing and MIC determinations of tigecycline are critical to clinical practice as well as for ongoing surveillance programs.

In many countries, agar dilution or broth microdilution method is recommended, because the tigecycline microdilution panel is still difficult to obtain on a large scale. The E-test strip can be set up as easily as a disc diffusion test by most clinical laboratories without the need for specialized equipment. The disc diffusion data should be supported by broth microdilution tests and further studies should be conducted to minimize false-susceptible errors. It is also important to decide the evaluation criteria to determine the antibiotic susceptibility properly. Interpretive breakpoints for susceptibility reporting by clinical microbiology laboratories were previously set for an antimicrobial agent with no consideration of bacterial species differences. In recent years such differences have been appreciated and species-related interpretive breakpoints are issued more frequently. Moreover, further studies are needed to define the most adequate methods for testing tigecycline susceptibility in Acinetobacter sp.

\section{Literature}

Bolmstrom A., A. Karlsson, A. Engelhardt, P. Ho, P.J. Petersen, P.A. Bradford and C.H. Jones. 2007. Validation and reproducibility assessment of tigecycline MIC determinations by etest. J. Clin. Microbiol. 45: 2474-2479.

Clinical and Laboratory Standards Institute. 2011. Performance antimicrobial susceptibility testing, Documents: M100-S16. CLSI. Wayne, PA. 
Falagas M.E., E.A. Karveli, I.I. Siempos and K.Z. Vardakas. 2008. Acinetobacter infections: a growing threat for critically ill patients. Epidemiol. Infect. 136: 1009-1019.

Fraise A.P. 2006. Tigecycline: The answer to beta-lactam and fluoroquinolone resistance? J. Infect. 53: 293-300.

Gülhan B., Ş. Nergiz, S. Meşe, T. Özekinci and S. Atmaca. 2009. Evaluation of disk diffusion zone diameters by two different breakpoints for tigecycline in Acinetobacter baumannii strains (in Turkish). ANKEM Derg. 23: 78-81.

Hope R., T. Parsons, S. Mushtaq, D. James and D.M. Livermore. 2007. Determination of disc breakpoints and evaluation of Etests for tigecycline susceptibility testing by the BSAC method. J. Antimicrob. Chemother. 60: 770-774.

Jones R.N., M.J. Ferraro, L. Barth Reler, P.C. Schreckenberger, J.M. Swenson and H.S. Sader. 2007. Multicenter studies of tigecycline disk diffusion susceptibility results for Acinetobacter spp. J. Clin. Microbiol. 45: 227-230.

Kulah C., G. Celebi, E. Aktas, Z. Mengeloglu, F. Comert and H. Ankarali. 2009. Unexpected tigecycline resistance among Acinetobacter baumannii Isolates: high minor error rate by E-test. J. Chemother. 21: 390-395.

Liu J.-W., T.-N. Jang, Y.-J. Cheng, G.-J. Hsu, W. Sun, C.-T. Lu and P.-R Husueh. 2010. Comparison of the E-test and broth microdilution method for tigecycline susceptibility testing against clinical isolates of Acinetobacter baumannii from Taiwan. Int. J. Antimic. Agents. 35: 200-209.

Manchanda V., S. Sanchaita and N.P. Singh. 2010. Multidrug resistant Acinetobacter. J. Global. Infect. Dis. 2: 291-304.
Mendes R.E., D.J. Biedenbach, M.R.K. Alley, H.S. Sader and R.N. Jones. 2010. Potency and spectrum of activity of AN 3365 a novel boron-containing protein synthesis inhibitor tested against Enterobacteriaceae $50^{\text {th }}$ ICAAC, Boston F1-1638.

Neonakis I.K., D.A. Spandidos and E. Petinaki. 2011. Confronting multidrug-resistant Acinetobacter baumannii: a review. Int. J. Antimic. Agents. 37: 102-109.

Peterson L.R. 2008. A review of tigecycline-the first glycylcycline. Int. J. Antimic. Agents. 32: 215-222.

Pillar C.M., D.C. Draghi, M.J. Dowzicky and D.F. Sahm. 2008. In vitro activity of tigecycline against Gram-positive and Gramnegative pathogens as evaluated by broth microdilution and Etest. J. Clin. Microbiol. 46: 2862-2867.

Shakoor S., E. Khan, A. Zafar and R. Hasan. 2011. In vitro activity of tigecycline and other tetracyclines against carbapenem-resistant Acinetobacter species: report from a tertiary care centre in Karachi, Pakistan. Chemotherapy 2010. 56:184-189.

Thamlikitkul V., S. Tiengrim and C. Tribuddharat. 2007. Comment on: High tigecycline resistance in multidrug-resistant Acinetobacter baumannii. J. Antimicrob. Chemother. 60: 177-178.

Wang Y.F. and M.J. Dowzicky. 2010. In vitro activity of tigecycline and comparators on Acinetobacter spp. isolates collected from patients with bacteremia and MIC change during the tigecycline evaluation and surveillance trial, 2004 to 2008. Diagn. Microbiol. Infect. Dis. 68: 73-79.

Zarate M.S., G. Serruto and J. Smayevsky. 2010. The susceptibility to tigecycline of Acinetobacter spp. may vary depending on the methodology used. Int. J. Inf. Dis. 14: 351-352. 